\title{
Solubility of some novel cyanopyridine derivatives
}

\begin{abstract}
Some new cyano pyridine derivatives have been synthesized and their characterization was done by IR, 1H NMR and mass spectral data. The solubility of these synthesized compounds has been studied in dimethyl formamide and dimethyl sulphoxide at different temperatures at atmospheric pressure.
\end{abstract}

Keywords: cyano pyridine derivative, solubility, DMF, DMSO, thermodynamic parameter
Volume 2 Issue 2 - 2018

Shipra Baluja, Jadgish Movalia

Department of Chemistry, Saurashtra University, India

Correspondence: Shipra Baluja, Department of Chemistry, Saurashtra University, Rajkot- 360 005, Gujarat, India, Email shipra_baluja@rediffmail.com

Received: April 03, 2018| Published: April 16, 2018
Abbreviations: DMF, dimethyl formamide; DMSO, dimethyl sulfoxide; RMSD, root-mean-square deviations; RD, relative deviations; RAD, relative average deviations

\section{Introduction}

Pyridine compounds exist in nature in various forms and are integral part of various natural products. ${ }^{1,2}$ The pyridine ring plays a key role in catalyzing both biological and chemical reactions. ${ }^{3}$ Various substituted pyridines demonstrate a wide range of applications. Among various substituted pyridines, cyano pyridine derivatives have been found to be an important sub class. Various substituted cyano pyridine derivatives are known to act as intermediates in the pharmaceutical, dye, photo and agrochemical industries. ${ }^{4-6}$ Further, various cyano pyridines have drawn attention due to their wide spectrum biological activities. $^{7-11}$ Therefore, due to their applications in biological and chemical fields, it would be interesting to determine the solubility of some novel cyano pyridine derivatives in different solvents at various temperatures. The data may be useful for their application in other fields also.

Thus, in the present work, solubility of some newly synthesized cyano pyridine derivatives is determined in dimethyl formamide and dimethyl sulfoxide at different temperatures. Further, some thermodynamic parameters such as enthalpy, Gibb's free energy and entropy of dissolution for these synthesized compounds have also been evaluated.

\section{Experimental}

The solvents dimethyl formamide (DMF) and dimethyl sulfoxide (DMSO) were used for the present study were purified by standard methods. ${ }^{12}$ All the synthesized compounds were crystallized and Figure 1 shows the general structure of these derivatives.

\section{Solubility}

The gravimetric method was used to study the solubility. An excess mass of compound was added to a known mass of solvent. The solution was heated to a constant temperature with continuous stirring. After, at least $3 \mathrm{hrs}$ the stirring was stopped and the solution was kept at a constant temperature for $2 \mathrm{hrs}$. A portion of this solution was filtered and by a preheated injector, $5 \mathrm{ml}$ of this clear solution was taken to pre weighted measuring vial $\left(\mathrm{m}_{0}\right)$. The vial was quickly and tightly closed and weighted $\left(\mathrm{m}_{1}\right)$ to determine the mass of the sample $\left(m_{1}-m_{0}\right)$. To prevent dust contamination, the vial was covered with a piece of filter paper. After completely dryness of vial mass, the vial was reweighed $\left(\mathrm{m}_{2}\right)$ to determine the mass of the constant residue solid $\left(\mathrm{m}_{2}-\mathrm{m}_{0}\right)$. All the weights taken using Mettler Toledo AB204-S, Switzerland electronic balance with uncertainty of $\pm 0.0001 \mathrm{~g}$. Thus the concentration of solid sample in the solution, mole fraction $\mathrm{x}$, could be determined from equation

$$
x=\frac{\left(m_{2}-m_{0}\right) / M_{1}}{\left(m_{2}-m_{0}\right) / M_{1}+\left(m_{1}-m_{2}\right) / M_{2}}
$$

Where $M_{1}$ and $M_{2}$ is the molar mass of solvent and compound respectively. At each temperature, the measurement was repeated three times and an average value is taken.

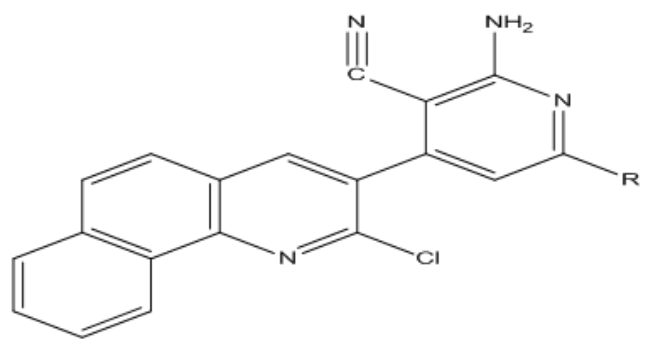

Figure I General structure of cyanopyridine derivatives.

Where $\mathrm{R}$ is:

$\mathrm{CP}-\mathrm{I}=4-\mathrm{OCH} 3 ; \mathrm{CP}-2=4-\mathrm{CH} 3 ; \mathrm{CP}-3=4-\mathrm{Br} ; \mathrm{CP}-4=4-\mathrm{NH} 2 ; \mathrm{CP}-5=$

4-NO2; $\mathrm{CP}-6=3-\mathrm{OH} ; \mathrm{CP}-7=4-\mathrm{Cl} ; \mathrm{CP}-8=3-\mathrm{NO} 2 ; \mathrm{CP}-9=4-\mathrm{OH} ; \mathrm{CP}-10=\mathrm{H}$

\section{Results and discussion}

The molecular formula, molecular weight, melting point, $\%$ yield and $R_{f}$ values along with the solvent systems of all the compounds are given in Table 1.

Table 2 and Table 3 show the experimental solubility values of compounds at different temperatures in DMF and DMSO respectively. The variation of mole fraction solubility of compounds with temperature in DMF and DMSO is shown in Figure 2 and Figure 3 respectively. It is observed that in both the solvents, solubility increases with temperature. Further, comparison of solubility in both the solvents; DMF and DMSO shows that overall solubility is greater in DMSO than that in DMF. Thus, the solvent polarity plays an important role on the solubility of studied compounds. The dielectric constant of DMSO (46.6) is greater than that of DMF (36.71). However, there is 
very small variation is in their dipole moments (3.9 for DMSO and 3.86 for DMF). This suggests that dielectric constant of solvent plays an important role in dissolution for the studied compounds.

The temperature dependence of solubility was described by the modified Apelblat equation ${ }^{13,14}$

$$
\ln x=A+\frac{B}{T}+C \ln T \ldots \text { (2) }
$$

Where $\mathrm{T}$ is the absolute temperature, and $\mathrm{A}, \mathrm{B}$, and $\mathrm{C}$ are empirical constants. The values of these parameters are listed in Table 4 . The root-mean-square deviations (RMSD) are calculated using the following equation:

$$
\operatorname{RMSD}=\sqrt{\sum_{i=1}^{N} \frac{\left(x_{i}-x\right)^{2}}{N-1}}
$$

Where $\mathrm{N}$ is the number of experimental points and $\mathrm{x}$ and $\mathrm{x}$ represent the mole fraction solubility of the experiment and that calculated from eq 2, respectively. These values are given in Table 4. Further, relative deviations (RD) and relative average deviations (RAD) are calculated by eq (4) and (5).

$$
\begin{aligned}
& R D=\left(\frac{x-x_{i}}{x}\right) \ldots \ldots \\
& \mathrm{RAD}=\frac{1}{N} \sum_{i}^{N} \frac{\left(x-x_{i}\right)}{x}
\end{aligned}
$$

Where $\mathrm{N}$ is the number of experimental points and $\mathrm{x}_{\mathrm{i}}$ is the solubility calculated by eq 2 . The values of relative deviation are listed in Table 2 and Table 3 for DMF and DMSO respectively and relative average deviation values are reported in Table 4 . It is evident from Table 2 and Table 3 that relative deviation (RD) values are not more than $1.85 \%$ for DMF and $2.86 \%$ for DMSO. Thus, there is good agreement between experimental and calculated solubility values in both the solvents. Using experimental data of solubility in different solvents, some thermodynamic parameters such as dissolution enthalpy, Gibb's energy of dissolution and entropy have also been evaluated. According to modified Van't Hoff equation ${ }^{15,16}$ the dissolution enthalpy $\left(\Delta H_{s o l}\right)$ were evaluated by following relation.

$$
\frac{\partial \ln x}{\partial\left(\frac{1}{T}-\frac{1}{T_{h m}}\right)}=-\frac{\Delta H_{\text {sol }}}{R} \ldots . .(6)
$$

Where $\mathrm{T}$ is the experimental temperature and $\mathrm{R}$ is universal gas constant. $\mathrm{T}_{\mathrm{hm}}$ represent the mean harmonic temperature which is given as

$$
T_{h m}=\frac{n}{\sum_{i}^{n}\left(\frac{1}{T}\right)} \ldots \ldots \text { (7) }
$$

Where $\mathrm{n}$ is the number of experimental temperatures. ${ }^{17}$ In present case, the value of $\mathrm{T}_{\text {hm }}$ is obtained only $308 \mathrm{~K}$. The slope of the plot of $\ln \mathrm{x}$ versus (1/T-1/308) gives the value of $\Delta \mathrm{H}_{\text {sol }}$. From the intercepts of these plots, Gibbs energy change $\left(\Delta \mathrm{G}_{\mathrm{sol}}\right)$ for dissolution process were calculated from the following relation ${ }^{15}$

$$
\Delta G_{s o l}=-R T_{h m} . \text { intercept }
$$

Using these evaluated $\Delta \mathrm{H}_{\text {sol }}$ and $\Delta \mathrm{G}_{\text {sol }}$ values, the entropies of solutions $\Delta \mathrm{S}_{\mathrm{sol}}$ were obtained from the following equation:

$$
\Delta S_{s o l}=\frac{\Delta H_{s o l}-\Delta G_{s o l}}{T_{h m}}
$$

All these thermodynamic parameters are listed in Table 5.

It is evident from Table 5, that for all the compounds, the evaluated thermodynamic parameters i.e., $\Delta \mathrm{H}_{\text {sol }}, \Delta \mathrm{G}_{\text {sol }}$ and $\Delta \mathrm{S}_{\text {sol }}$ values are positive for both the solvents. The positive $\Delta \mathrm{H}_{\text {sol }}$ suggests endothermic dissolution of compounds in both the solvents. The endothermic effect may be due to strong interactions between compound and solvent molecules. ${ }^{17,18}$ Whereas, positive $\Delta \mathrm{G}_{\text {sol }}$ values indicate that the

\begin{tabular}{|c|c|c|c|c|c|c|c|}
\hline Sr. No & Comp. code & Mol.Wt. g/mol) & M.F. & $\mathbf{R}$ & Rf* Value & M.P. ${ }^{\circ} \mathrm{C}$ & Yield \% \\
\hline I & CP-I & 436.8 & $\mathrm{C}_{27} \mathrm{H}_{17} \mathrm{CIN}_{4} \mathrm{O}$ & $4-\mathrm{OCH}_{3}-\mathrm{C}_{6} \mathrm{H}_{4}-$ & 0.59 & 221 & 70 \\
\hline 2 & CP -2 & 420.8 & $\mathrm{C}_{26} \mathrm{H}_{17} \mathrm{CIN}_{4}$ & $4-\mathrm{CH}_{3}-\mathrm{C}_{6} \mathrm{H}_{4}^{-}$ & 0.56 & 180 & 68 \\
\hline 3 & $\mathrm{CP}-3$ & 485.7 & $\mathrm{C}_{25} \mathrm{H}_{14} \mathrm{BrClN}_{4}$ & $4-\mathrm{Br}-\mathrm{C}_{6} \mathrm{H}_{4^{-}}^{-}$ & 0.63 & 214 & 71 \\
\hline 4 & CP -4 & 421.8 & $\mathrm{C}_{25} \mathrm{H}_{16} \mathrm{CIN}_{5}$ & 4- $\mathrm{NH}_{2}-\mathrm{C}_{6} \mathrm{H}_{4}^{-}$ & 0.69 & 208 & 65 \\
\hline 5 & CP -5 & 451.8 & $\mathrm{C}_{25} \mathrm{H}_{14} \mathrm{CIN}_{5} \mathrm{O}_{2}$ & $4-\mathrm{NO}_{2}-\mathrm{C}_{6} \mathrm{H}_{4}^{-}$ & 0.64 & 187 & 69 \\
\hline 6 & CP -6 & 422.8 & $\mathrm{C}_{25} \mathrm{H}_{15} \mathrm{CIN}_{4} \mathrm{O}$ & $3-\mathrm{OH}-\mathrm{C}_{6} \mathrm{H}_{4}^{-}$ & 0.7 & 235 & 67 \\
\hline 7 & CP -7 & 441.3 & $\mathrm{C}_{25} \mathrm{H}_{14} \mathrm{Cl}_{2} \mathrm{~N}_{4}$ & $4-\mathrm{Cl}-\mathrm{C}_{6} \mathrm{H}_{4^{-}}$ & 0.72 & 234 & 72 \\
\hline 8 & CP -8 & 451.8 & $\mathrm{C}_{25} \mathrm{H}_{14} \mathrm{ClN}_{5} \mathrm{O}_{2}$ & $3-\mathrm{NO}_{2}-\mathrm{C}_{6} \mathrm{H}_{4}^{-}$ & 0.62 & 201 & 63 \\
\hline 9 & CP -9 & 422.8 & $\mathrm{C}_{25} \mathrm{H}_{15} \mathrm{CIN}_{4} \mathrm{O}$ & $4-\mathrm{OH}-\mathrm{C}_{6} \mathrm{H}_{4}^{-}$ & 0.67 & 229 & 65 \\
\hline 10 & $C P-10$ & 406.8 & $\mathrm{C}_{25} \mathrm{H}_{15} \mathrm{ClN}_{4}$ & $\mathrm{H}-\mathrm{C}_{6} \mathrm{H}_{4}^{-}$ & 0.49 & 162 & 73 \\
\hline
\end{tabular}
dissolution process is spontaneous. The positive entropy indicates that dissolution process increases the randomness in solution. ${ }^{18}$ However, for some compounds entropy is less than half value than those of other compounds. This depends on the functional groups present in the compound as well as on the solvent. Different functional groups interact differently with the solvent, so randomness will be different.

Table I Physical constant of Cyano pyridine compounds

*Ethyl acetate: Hexane: 3:7 


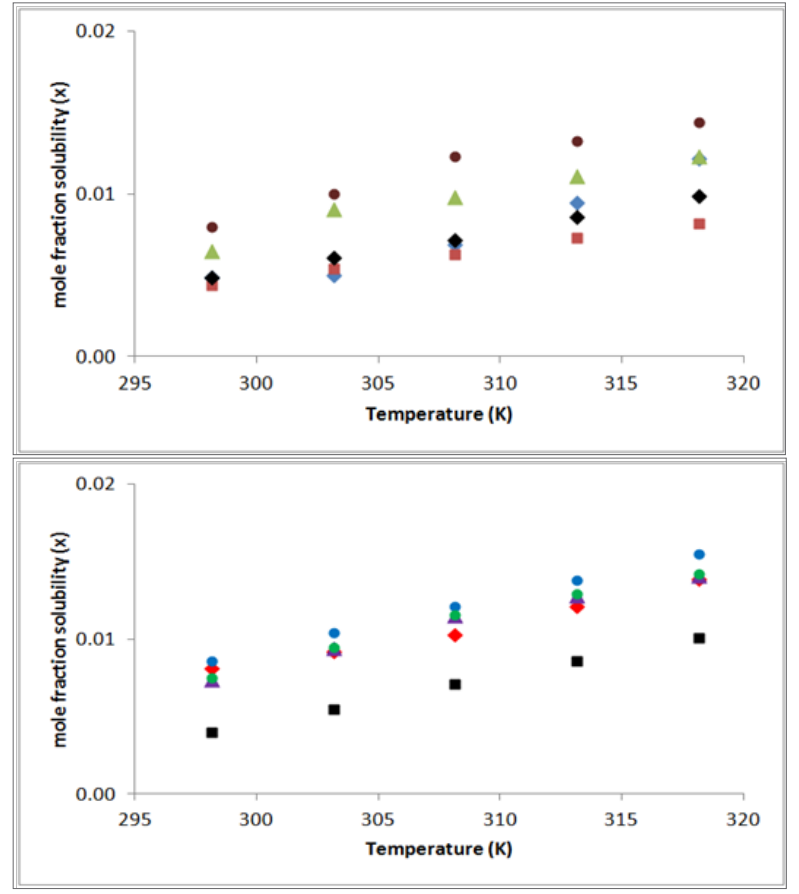

Figure 2 The variation of experimental mole fraction solubility $(x)$ of compounds with temperature in DMF.

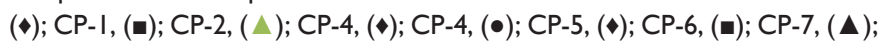

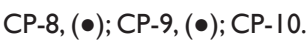

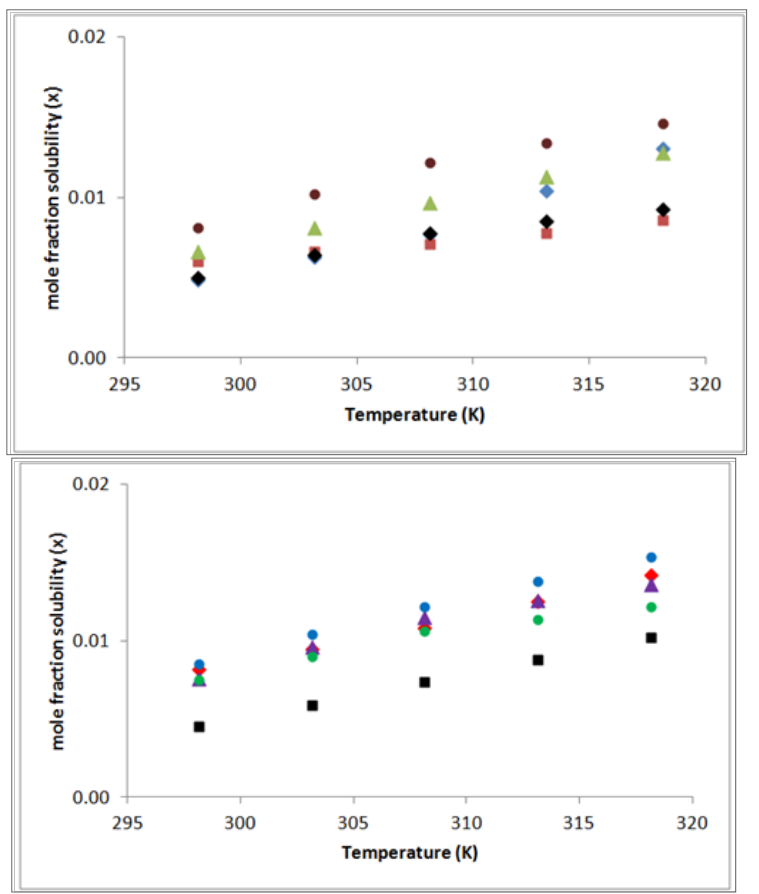

Figure 3 The variation of experimental mole fraction solubility $(x)$ of compounds with temperature in DMSO.

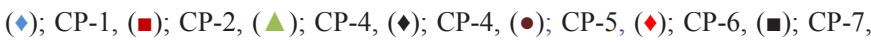
$(\mathbf{\Delta})$; CP-8, (•); CP-9, (•); CP-10.

Table 2 The experimental solubility (x), calculated solubility $(x c)$ and relative deviation (RD) of anopyridines derivatives in DMF at different temperatures.

\begin{tabular}{|c|c|c|c|c|c|c|}
\hline Temp.K & $\mathbf{x}$ & $x_{c}$ & I 00 RD & $\mathbf{x}$ & $x_{c}$ & I 00 RD \\
\hline & CP-I & & & CP-6 & & \\
\hline 298.15 & 0.0049 & 0.004873 & 0.553 & 0.0081 & 0.008117 & -0.2144 \\
\hline 303.15 & 0.0055 & 0.005593 & -1.6838 & 0.0092 & 0.00915 & 0.5447 \\
\hline 308.15 & 0.0069 & 0.006858 & 0.6078 & 0.0103 & 0.010427 & -1.2285 \\
\hline 313.15 & 0.009 & 0.008947 & 0.5884 & 0.0121 & 0.012002 & 0.8086 \\
\hline \multirow[t]{2}{*}{318.15} & 0.0123 & 0.012368 & -0.5563 & 0.0139 & 0.013947 & -0.3354 \\
\hline & CP-2 & & & CP-7 & & \\
\hline 298.15 & 0.0044 & 0.004415 & -0.3523 & 0.004 & 0.004012 & -0.2966 \\
\hline 303.15 & 0.0054 & 0.005367 & 0.6156 & 0.0055 & 0.005488 & 0.2174 \\
\hline 308.15 & 0.0063 & 0.006343 & -0.6819 & $0.007 \mid$ & 0.007087 & 0.1892 \\
\hline 313.15 & 0.0073 & 0.007302 & -0.0298 & 0.0086 & 0.008668 & -0.7937 \\
\hline \multirow[t]{2}{*}{318.15} & 0.0082 & 0.008201 & -0.0139 & 0.0101 & 0.010077 & 0.2285 \\
\hline & CP-3 & & & CP-8 & & \\
\hline 298.15 & 0.0065 & 0.0065 & $0.005 \mathrm{I}$ & 0.0074 & 0.007406 & -0.0775 \\
\hline 303.15 & 0.0081 & $0.008|4|$ & -0.5025 & 0.0094 & 0.009446 & -0.4843 \\
\hline 308.15 & 0.0098 & 0.00974 & 0.6075 & 0.0115 & 0.011371 & 1.1229 \\
\hline 313.15 & 0.0111 & 0.011165 & -0.5862 & 0.0128 & 0.012966 & -1.2995 \\
\hline
\end{tabular}


Table Continued...

\begin{tabular}{lllllll}
\hline Temp.K & $\mathbf{x}$ & $\mathbf{x}_{\mathbf{c}}$ & $\mathbf{1 0 0} \mathbf{R D}$ & $\mathbf{x}$ & $\mathbf{x}_{\mathbf{c}}$ & I00 RD \\
\hline 318.15 & 0.0123 & 0.012292 & 0.061 & 0.0141 & 0.014052 & 0.3405 \\
\hline & $\mathbf{C P}-4$ & & & $\mathbf{C P}-9$ & & \\
\hline 298.15 & 0.0049 & 0.004919 & -0.3924 & 0.0086 & 0.008626 & -0.3023 \\
303.15 & 0.0061 & 0.006049 & 0.8295 & 0.0104 & 0.010358 & 0.4006 \\
308.15 & 0.0072 & 0.007277 & -1.0755 & 0.0121 & 0.012125 & -0.2068 \\
313.15 & 0.0086 & 0.008575 & 0.285 & 0.0138 & 0.013857 & -0.4096 \\
318.15 & 0.0099 & 0.00991 & -0.102 & 0.0155 & 0.015482 & 0.1165 \\
\hline & $\mathbf{C P - 5}$ & & & $\mathbf{C P}-10$ & & \\
\hline 298.15 & 0.008 & 0.008006 & -0.0787 & 0.0075 & 0.007506 & -0.0787 \\
303.15 & 0.0101 & 0.010167 & -0.6589 & 0.0095 & 0.009547 & -0.4976 \\
308.15 & 0.0123 & 0.012098 & 1.6408 & 0.0116 & 0.011471 & 1.1087 \\
313.15 & 0.0133 & 0.013546 & -1.851 & 0.0129 & 0.013066 & -1.2862 \\
318.15 & 0.0144 & 0.014325 & 0.5219 & 0.0142 & 0.014154 & 0.3261
\end{tabular}

Table 3 The experimental solubility $(\mathrm{x})$, calculated solubility $(\mathrm{xc})$ and relative deviation $(\mathrm{RD})$ of cyanopyridines derivatives in DMSO at different temperatures.

\begin{tabular}{|c|c|c|c|c|c|c|}
\hline Temp.K & $\mathbf{x}$ & $x_{c}$ & 100 RD & $x$ & $x_{c}$ & 100 RD \\
\hline & CP-I & & & CP-6 & & \\
\hline 298.15 & 0.0049 & 0.004919 & -0.3816 & 0.0082 & 0.008206 & -0.0724 \\
\hline 303.15 & 0.0063 & 0.006213 & |.377| & 0.0095 & 0.009474 & 0.2704 \\
\hline 308.15 & 0.0077 & 0.007921 & -2.8667 & 0.0108 & 0.01089 & -0.8342 \\
\hline 313.15 & 0.0104 & 0.010183 & 2.0845 & 0.0125 & 0.012464 & 0.2849 \\
\hline \multirow[t]{2}{*}{318.15} & 0.0131 & 0.013194 & -0.7213 & 0.0142 & 0.014209 & -0.0606 \\
\hline & CP-2 & & & CP-7 & & \\
\hline 298.15 & 0.006 & 0.005988 & 0.193 & 0.0045 & 0.004508 & -0.1743 \\
\hline 303.15 & 0.0066 & 0.00657 I & 0.4398 & 0.0059 & 0.005902 & -0.038 \\
\hline 308.15 & 0.0071 & 0.007189 & -1.259 & 0.0074 & 0.007384 & 0.2207 \\
\hline 313.15 & 0.0078 & 0.007844 & -0.5691 & 0.0088 & 0.00885 & -0.5667 \\
\hline \multirow[t]{2}{*}{318.15} & 0.0086 & 0.008537 & 0.737 & 0.0102 & 0.010189 & 0.1077 \\
\hline & CP-3 & & & CP-8 & & \\
\hline 298.15 & 0.0066 & $0.00660 \mathrm{I}$ & -0.0213 & 0.0076 & 0.007617 & -0.2262 \\
\hline 303.15 & 0.0081 & 0.008119 & -0.2378 & 0.0096 & 0.00961 & -0.1085 \\
\hline 308.15 & 0.0097 & 0.009706 & -0.058 & 0.0115 & 0.011402 & 0.8531 \\
\hline 313.15 & 0.0113 & 0.011295 & 0.0408 & 0.0126 & 0.012769 & -1.3403 \\
\hline \multirow[t]{2}{*}{318.15} & 0.0128 & 0.012819 & -0.1456 & 0.0136 & 0.013546 & 0.3978 \\
\hline & CP-4 & & & CP-9 & & \\
\hline 298.15 & 0.005 & 0.005014 & -0.2731 & 0.0085 & 0.008526 & -0.3015 \\
\hline 303.15 & 0.0064 & $0.0064 I 4$ & -0.2132 & 0.0104 & 0.010368 & 0.3039 \\
\hline 308.15 & 0.0078 & 0.007689 & 1.4236 & 0.0122 & 0.012193 & 0.0572 \\
\hline 313.15 & 0.0085 & 0.008673 & -2.0393 & 0.0138 & 0.013894 & -0.6778 \\
\hline \multirow[t]{2}{*}{318.15} & 0.0093 & 0.00924 & 0.6421 & 0.0154 & 0.015369 & 0.202 \\
\hline & CP-5 & & & CP-IO & & \\
\hline 298.15 & 0.0081 & 0.008122 & -0.2738 & 0.0075 & 0.007495 & 0.0622 \\
\hline 303.15 & 0.0102 & 0.010198 & 0.0151 & 0.009 & 0.009075 & $-0.834 I$ \\
\hline
\end{tabular}


Table Continued...

\begin{tabular}{lllllll}
\hline Temp.K & $\mathbf{x}$ & $\mathbf{x}_{\mathbf{c}}$ & I00 RD & $\mathbf{x}$ & $\mathbf{x}_{\mathbf{c}}$ & I00 RD \\
\hline 308.15 & 0.0122 & 0.012095 & 0.864 & 0.0106 & 0.010466 & 1.2654 \\
313.15 & 0.0134 & 0.013595 & -1.4544 & 0.0114 & 0.011531 & -1.1507 \\
318.15 & 0.0146 & 0.014532 & 0.4683 & 0.0122 & 0.012172 & 0.2297 \\
\hline
\end{tabular}

Table 4 Coefficients A, B and C of equation 2, relative average deviation (RAD) and root mean square deviation (rmsd) of cyanopyridine derivatives in DMF and DMSO.

\begin{tabular}{|c|c|c|c|c|c|c|}
\hline Compounds & A & B & C & $\gamma$ & $10^{5} \mathrm{rmsd}$ & $10^{2}$ RAD \\
\hline & DMSO & & & & & \\
\hline CP-I & -1768.58 & 76932.79 & 254.9775 & 0.989 & 17.7 & -0.1127 \\
\hline CP-2 & 548.9647 & -2785 I & -80.907 & 0.9998 & 2.83 & -0.0925 \\
\hline CP-3 & 971.1418 & -47221.7 & -143.534 & 0.9998 & 4.87 & -0.083 \\
\hline $\mathrm{CP}-4$ & 390.0745 & -20902.7 & -57.0911 & 0.9997 & 4.91 & -0.0911 \\
\hline CP-5 & 1442.436 & -68552.3 & -213.658 & 0.9985 & 16.69 & -0.0852 \\
\hline $\mathrm{CP}-6$ & -367.517 & |442|.| | & 55.16949 & 0.9993 & 8.74 & -0.085 \\
\hline CP-7 & $|204.3| 8$ & $-5905 I .6$ & -177.58 & 0.9999 & 3.78 & -0.0911 \\
\hline CP-8 & 1259.862 & -60438.5 & -186.404 & 0.9994 & 11.04 & -0.0796 \\
\hline CP-9 & 497.3518 & -25321.4 & -73.2199 & 0.9999 & 4.05 & -0.0803 \\
\hline \multirow[t]{2}{*}{ CP- 10} & $|24| .62 \mid$ & -59578.3 & -183.707 & 0.9994 & 11.01 & -0.0856 \\
\hline & DMSO & & & & & \\
\hline CP-I & -410.769 & -14588 & 62.57469 & 0.9988 & 16.77 & -0.1016 \\
\hline CP-2 & -2.57231 & -1540.24 & 0.459747 & 0.9984 & 6.11 & -0.0917 \\
\hline CP-3 & 553.1926 & -28204.6 & -81.3703 & 0.9999 & 1.39 & -0.0844 \\
\hline CP-4 & 1435.946 & -68395.8 & -212.693 & 0.9987 & 10.76 & -0.092 \\
\hline CP-5 & 1255.235 & -59986.6 & -185.842 & 0.9993 & 11.65 & -0.0762 \\
\hline $\mathrm{CP}-6$ & -1.28015 & -2365.81 & 0.774249 & 0.9998 & 5.04 & -0.0824 \\
\hline CP-8 & $|36| .63 \mid$ & -64833.1 & -201.674 & 0.9994 & 10.18 & -0.0848 \\
\hline CР-9 & 689.6105 & $-34 \mid 35.8$ & -101.777 & 0.9999 & 5.34 & -0.0832 \\
\hline CP- 10 & 1070.677 & -51157.2 & -158.662 & 0.9988 & 10.2 & -0.0855 \\
\hline
\end{tabular}

Table 5 Thermodynamic parameters of dissolution of compounds in DMF and DMSO.

\begin{tabular}{|c|c|c|c|c|c|c|}
\hline Comp.code & $\Delta \mathbf{H}_{\text {sol }} \mathbf{~ k J . m o l}{ }^{-1}$ & $\Delta \mathbf{G}_{\text {sol }} \mathbf{~ k J . m o l}{ }^{-1}$ & $\Delta \mathbf{S}_{\text {sol }} \mathbf{J} \cdot \mathrm{mol}^{-1} \cdot \mathbf{K}^{-1}$ & $\Delta \mathbf{H}_{\text {sol }} \mathbf{~ k J . m o l}{ }^{-1}$ & $\Delta \mathbf{G}_{\text {sol }} \mathbf{k J} \cdot \mathbf{m o l}^{-1}$ & $\Delta \mathbf{S}_{\text {sol }} \mathbf{J} \cdot \mathbf{m o l}^{-1} \cdot \mathbf{K}^{-1}$ \\
\hline & DMF & & & DMSO & & \\
\hline CP-I & 39.01 & 12.63 & 85.64 & 39.15 & 12.37 & 86.94 \\
\hline CP -2 & 24.25 & 13.02 & 36.47 & 13.88 & 12.65 & 41 \\
\hline CP -3 & 25.33 & 11.97 & 43.38 & 26.4 & 11.94 & 46.94 \\
\hline CP -4 & 27.5 & 12.65 & 48.19 & 23.91 & 12.62 & 36.65 \\
\hline CP -5 & 22.75 & 11.46 & 36.65 & 22.71 & 11.44 & 36.58 \\
\hline CP -6 & 21.18 & 11.66 & 30.93 & 21.47 & 11.59 & 32.08 \\
\hline CP -7 & 36.22 & $|2.8|$ & 75.98 & 32.59 & 12.69 & 64.64 \\
\hline CP -8 & 25.46 & 11.6 & 44.98 & 22.98 & 11.61 & 36.91 \\
\hline CP -9 & 22.85 & 11.36 & 37.32 & 23.23 & 11.36 & 38.52 \\
\hline$C P-10$ & 25.11 & 11.58 & 43.92 & 19.08 & 11.8 & 23.66 \\
\hline
\end{tabular}




\section{Conclusion}

It is concluded that solubility increases with temperature in both the solvents. Overall, solubility is greater in DMSO than that in DMF for all the compounds. Further, the evaluated thermodynamic parameters i.e., enthalpy, Gibb's free energy and entropy of dissolutions values are positive for both the solvents. The positive enthalpy suggests endothermic dissolution of compounds in both the solvents indicating thereby strong interactions between compound and solvent molecules. The positive Gibb's free energy and entropy indicate that dissolution process is spontaneous and it increases the randomness in solution.

\section{Acknowledgments}

Authors are thankful to Head of Chemistry Department, Saurashtra University, Rajkot, India for providing necessary facilities.

\section{Conflict of inertest}

There is no conflict of interest.

\section{References}

1. Scriven EVF. Pyridines: from Lab to Production, Ist ed. Amsterdam Elsevier; 2013.

2. Schlosser M, Mongin F. Pyridine elaboration through organometallic intermediates: region chemical control and completeness. Chem Soc Rev. 2007;36(7):1161-1172.

3. Chaubey A, Pandaya SN. Pyridine: A versatile nuclease in pharmaceutical field. Asian J Pharma Clin Res. 2011;4(4):5-8.

4. You J, Lai SL, Liu W, et al. Bipolar cyano-substituted pyridine derivatives for applications in organic light-emitting devices. $J$ Mater Chem. 2012;22(18):8922-8929.

5. Oganisyan S, Noravyan AS, Grigoryan MZ. Condensed pyridopyrimidines.7. Synthesis of condensed triazolo[4,3-c]- and tetrazolo[1,5-] pyrimidnes. Chem Heterocyclic Compds. 2004;40(1):75-78.

6. Bowman MD, Jacobson MM, Blackwell HE. Discovery of Fluorescent Cyanopyridine and Deazalumazine Dyes Using Small Molecule Macroarrays. Org Lett. 2006;8(8):1645-1648.
7. Bernardino MR, LC da S Pinheiro PG, Rodrigues $\mathrm{CR}$, et al. Design, synthesis, SAR, and biological evaluation of new 4-(phenylamino)thieno[2,3-b] pyridine derivatives. Bioorg Med Chem. 2006;14(16):5765-5770.

8. Márquez MJ, Márquez MB, Cataldo PG, et al. A Comparative Study on the Structural and Vibrational Properties of Two Potential Antimicrobial and Anticancer Cyanopyridine Derivatives. Open J Syn Theory Appl. 2015;4(1):1-19.

9. Saad HA, Mokbil MN, El-Gendy AM, et al. Synthesis of some glycosides of pyridinone derivatives. Synth Commun. 2002;32(8):1189-1195.

10. Dolle V, Fan E, Nguyen $\mathrm{CH}$, et al. A new series of pyridinone derivatives as potent non-nucleoside human immuno deficiency virus type 1 specific reverse transcriptase inhibitors. J Med Chem. 1995;38(23):4679-4686.

11. Sondhi SM, Jain S, Dinodia M, et al. Synthesis of some thiophenes, imidazole and pyridine derivatives exhibiting good anti-inflammatory and analgesic activities. Med Chem. 2008;4(2):146-154.

12. Riddick JA, Bunger WB, Sakano TK. Organic Solvents-Physical Properties and Methods of Purification. 4th ed. Techniques of Chemistry. New Jersey: John Wiley; 1986. p. 1131-1294.

13. Hao HX, Wang JK, Wang YL. Solubility of dexamethasonesodium phosphate in different solvents. JChem Eng Data. 2004;49(6):1697-1698.

14. Nie Q, Wang JK, Wang YL. Solubility of $11 \alpha$-hydroxy-16 $\alpha, 17 \alpha$ Eepoxyprogestrone in different solvents between 283 and 323K. J Chem Eng Data. 2005;50:989-992.

15. Krug RR, Hunter WG, Grieger RA. Enthalpy entropy compensation. 2. Separation of the Chemical from the Statistical Effects. J Phys Chem. 1976;80(21):2341-2351.

16. Bustamante SP, Romero AP, Escalera B, et al. Nonlinear enthalpyEntropy Compensation for the Solubility of Drugs in Solvent Mixtures: Paracetamol, Acetanilide and Nalidixic acid in dioxane-water. J Pharma Sci. 1998;87(12):1590-1596.

17. Aragon DM, Ruidiaz MA, Vargas EF, et al. Solubility of the Antimicrobial Agent Triclosan in Organic Solvents of Different Hydrogen Bonding Capabilities at Several Temperatures. J Chem Eng Data. 2008;53(11):2576-2580.

18. Kalsi PS. Organic reactions and their mechanisms. 2nd ed. New Delhi: New age international (P) limited; 2004. p. 119. 